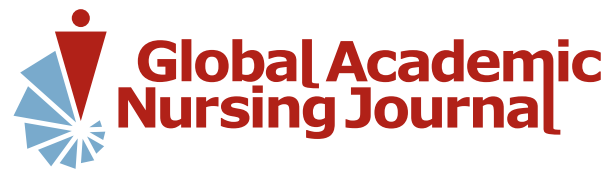

\section{Desenvolvimento da competência cultural na formação dos estudantes de enfermagem}

\author{
Development of cultural competence in the training of nursing students
}

Desarrollo de la competencia cultural en la formación de estudiantes de enfermería

Elisabete Aparecida Ribeiro Jose ${ }^{1}$
ORCID: 0000-0002-9980-4564

Ana Margarida da Silva Ribeiro Martins Coelho²

ORCID: 0000-0003-2531-4447

Magali Cruz Gouveia ${ }^{2}$

ORCID: 0000-0002-1410-3313

Marta Gomes Paiva ${ }^{2}$

ORCID: 0000-0002-2264-0485

Irma da Silva Brito ${ }^{2}$

ORCID: 0000-0002-8825-4923

Lina Márcia Migueis Berardinelli ${ }^{3}$

ORCID: 0000-0002-9481-8414

Corália Maria Fortuna de Brito

Vicente $^{1}$

ORCID: 0000-0002-2082-4483

1Universidade do Porto. Porto, Portugal.

2Escola Superior de Enfermagem de Coimbra. Coimbra, Portugal.

3Universidade Federal do Estado do Rio de Janeiro. Rio de Janeiro, Brasil.

\section{Como citar este artigo:}

Jose EAR, Coelho AMSRM, Gouveia

MC, Paiva MG, Brito IS, Berardinelli

LMM, Vicente CMFB.

Desenvolvimento da competência cultural na formação dos estudantes de enfermagem. Glob Acad Nurs. 2021;2(3):e154.

https://dx.doi.org/10.5935/26755602.20200154

\section{Autor correspondente:}

Elisabete Aparecida Ribeiro Jose

E-mail: dr.elisabeteadv@gmail.com

Editor Chefe: Caroliny dos Santos Guimarães da Fonseca

Editor Executivo: Kátia dos Santos Armada de Oliveira

Submissão: 02-08-2021

Aprovação: 19-10-2021

\section{Resumo}

Objetivou-se identificar e mapear as estratégias de desenvolvimento da competência cultural durante a formação dos estudantes nas instituições de ensino de enfermagem. Para a elaboração da presente Revisão Integrativa da Literatura, recorreu-se ao método PCC, de forma a desenvolver a questão de investigação: Quais são as estratégias utilizadas pelas Instituições de Ensino de Enfermagem para Desenvolvimento da competência cultural durante a formação dos estudantes? De modo a dar resposta à questão formulada, realizou-se uma pesquisa, com recurso aos motores de busca EBSCO-host e PubMed, e às respectivas bases de dados Cumulative Index to Nursing and Allied Health Literature e MedLine. Não foram aplicados limites de horizonte temporal. Os idiomas estabelecidos foram, português, inglês e espanhol. Para a avaliação da qualidade metodológica, utilizamos um instrumento de avaliação crítica, específico para cada tipo de estudo (qualitativo e quantitativo). Sete estudos foram incluídos na seleção, que após analisados demonstraram que há estratégias através das quais os estudantes podem desenvolver CC, porém as mais utilizadas são: os Programas de Mobilidade e os Cursos de Competência Cultural. Estes resultados fornecem evidências do potencial da utilização das estratégias educativas relativamente ao desenvolvimento da CC nos estudantes de Enfermagem.

Descritores: Competência Cultural; Educação em Enfermagem; Educação Continuada; Comunicação para Apreensão de Informação; Análise e Desempenho de Tarefas.

\section{Abstract}

The aim was to identify and map the strategies for the development of cultural competence during the training of students in nursing education institutions. For the elaboration of this Integrative Literature Review, the PCC method was used to develop the research question: What are the strategies used by Nursing Education Institutions to develop cultural competence during the training of students? To answer the question asked, a search was carried out, using the EBSCO-host and PubMed search engines, and the respective databases Cumulative Index to Nursing and Allied Health Literature and MedLine. No time horizon limits were applied. The established languages were Portuguese, English and Spanish. For the assessment of methodological quality, we used a critical assessment instrument, specific for each type of study (qualitative and quantitative). Seven studies were included in the selection, which after being analyzed showed that there are strategies through which students can develop CC, but the most used are: Mobility Programs and Cultural Competence Courses. These results provide evidence of the potential use of educational strategies for the development of CC in nursing students.

Descriptors: Cultural Competency; Education, Nursing; Education, continuing; Teach-Back Communication; Task Performance and Analysis.

Resumén

El objetivo fue identificar y mapear las estrategias para el desarrollo de la competencia cultural durante la formación de estudiantes en instituciones de educación en enfermería. Para la elaboración de esta Revisión Integrativa de la Literatura se utilizó el método PCC, con el fin de desarrollar la pregunta de investigación: ¿Cuáles son las estrategias que utilizan las Instituciones de Educación en Enfermería para desarrollar la competencia cultural durante la formación de los estudiantes? Para dar respuesta a la pregunta planteada, se realizó una búsqueda, utilizando los motores de búsqueda EBSCO-host y PubMed, y las respectivas bases de datos Cumulative Index to Nursing and Allied Health Literature y MedLine. No se aplicaron límites de horizonte temporal. Los idiomas establecidos fueron portugués, inglés y español. Para la evaluación de la calidad metodológica se utilizó un instrumento de evaluación crítica, específico para cada tipo de estudio (cualitativo y cuantitativo). En la selección se incluyeron siete estudios, que luego de ser analizados mostraron que existen estrategias a través de las cuales los estudiantes pueden desarrollar CC, pero las más utilizadas son: Programas de Movilidad y Cursos de Competencia Cultural. Estos resultados evidencian el potencial uso de estrategias educativas para el desarrollo de CC en estudiantes de enfermería.

Descriptores: Competencia Cultural; Educación En Enfermería; Educación Continua; Método Teach-Back; Análisis y Desempeño de Tareas. 
Desenvolvimento da competência cultural na formação dos estudantes de enfermagem Jose EAR, Coelho AMSRM, Gouveia MC, Paiva MG, Brito IS, Berardinelli LMM, Vicente CMFB

\section{Introdução}

Competência Cultural (CC) é o processo em que o profissional de saúde se esforça continuamente para alcançar a capacidade de prestar um cuidado de saúde eficaz, tendo em conta o contexto cultural do cliente, entendido como a pessoa, a família ou a comunidade. Apesar desse conceito ser o mais utilizado na enfermagem, tem, nas últimas décadas, evoluído e sido adaptado decorrente das mudanças sociais associadas à diversidade cultural, às políticas de saúde, ao aumento da pobreza e à iniquidade de acesso à saúde ${ }^{1,2}$.

Insta importante destacar que a globalização e o crescente aumento do fluxo migratório em que o mundo está exposto, corroboram com a premente necessidade de os enfermeiros desenvolverem Competência Cultural de forma a assegurar o cuidado sob uma perspectiva holística, respeitando as dimensões biológica, cultural, social e espiritual da pessoa, família e comunidade. A invasão cultural em que os países estão expostos, advinda através do crescente fluxo migratório, impacta todos os seguimentos da sociedade, sobretudo o seguimento da saúde que tem como desafio, reduzir as disparidades e garantir acesso universal, integral e equitativo aos serviços e ações de saúde respeitando a cultura dos usuários do sistema de saúde, o que somente será possível através do desenvolvimento da competência cultural. Evidências demonstram que os impactos sociais, culturais e econômicos resultantes da globalização podem redundar em riscos à saúde $\mathrm{e}^{3-8}$.

Salienta-se que a globalização, o aumento do fluxo migratório, e essa invasão cultural, detém impacto também no setor educacional, sobretudo no Ensino de Enfermagem pois, a enfermagem é essencialmente uma profissão de cuidados transculturais e interculturais ${ }^{9}$. Nesse sentido, o desenvolvimento dessa Competência, durante a formação, permitirá ao futuro enfermeiro prestar cuidados à pessoa sob uma perspectiva holística, minimizando as disparidades, e eventuais conflitos, que surgem quando diferentes culturas se cruzam².

Desse modo, a ênfase na formação em saúde desprovida da formação cultural e vice-versa reduzem a qualidade dos cuidados e a eficácia dos cuidados e dos serviços de saúde, logo, é indiscutível a necessidade de introduzir o conhecimento em cuidados culturais na formação dos graduandos ${ }^{10}$.

As Instituições de Ensino Superior, em específico as Escolas de Enfermagem, tem uma enorme responsabilidade na formação e capacitação dos alunos, pois os enfermeiros são os profissionais que devem prestar cuidados de forma holística considerando a subjetividade de cada pessoa, sobretudo as que estão em processos de transição de vida, como é o caso dos imigrantes ${ }^{9}$. Portanto, as Instituições de ensino têm o desafio de reformular ou potencializar seu currículo educacional em consonância com as tendências globais propiciando ao aluno, aporte teórico e interações com diversas culturas com ou sem problemas de saúde, visto que, os processos formativos necessitam estar focados nesta problemática, pois a educação intercultural é uma proposta político-pedagógica que vislumbra a formação para a cidadania e a superação de preconceitos e discriminações que expropriam de seus direitos os indivíduos e as comunidades $^{11}$.

Desse modo, este estudo vem contribuir para fortalecer a formação dos estudantes de enfermagem através do desenvolvimento das Competência técnicocientíficas, ético políticas, socioeducativas contextualizadas que permitam ao futuro enfermeiro, reconhecer a saúde como direito e condições dignas de vida e atuar de forma a garantir a integralidade da assistência, entendida como conjunto articulado e contínuo das ações e serviços preventivos e curativos, individuais e coletivos, exigidos para cada caso em todos os níveis de complexidade do sistema ${ }^{12}$. Sendo assim, objetivou-se realizar essa revisão de literatura para identificar e mapear as evidências científicas sobre as estratégias de desenvolvimento da competência culturalque contribuem para a formação dos estudantes nas Instituições de Ensino de Enfermagem a fim de responder a seguinte pergunta: Quais são as estratégias utilizadas pelas Instituições de Ensino de Enfermagem para Desenvolvimento da competência cultural durante a formação dos estudantes?

\section{Metodologia}

A Revisão Integrativa de Literatura (RIL) é um método de pesquisa que consiste na colheita e síntese ordenada de dados acerca de um tema ou questão, permitindo a aquisição de conhecimentos sobre a temática em análise. Esta síntese de conhecimento sustentará a tomada de decisão e posteriormente a melhoria da prática clínica, constituindo um instrumento fulcral para o saber em enfermagem ${ }^{13}$. Para o processo de formulação de uma Revisão Sistemática da Literatura (RSL), é imprescindível executar algumas etapas, nomeadamente: a formulação de uma questão de revisão; de definição de critérios inclusão e exclusão; localização e seleção dos estudos e posterior avaliação da qualidade metodológica deles; extração de dados; análise e síntese dos resultados mais pertinentes dos estudos selecionados; apresentação, interpretação e aplicabilidade dos resultados obtidos ${ }^{14}$. Nesta linha de pensamento, o presente documento reger-se-á pelos pressupostos de uma RIL, utilizando a metodologia da Joanna Briggs Institute (JBI) ${ }^{15}$.

Assim, de modo a construir a questão de pesquisa, utilizamos a mnemónica PCC a qual compreende três componentes para o processo de revisão (População: Estudantes, Conceito: Estratégias de Desenvolvimento da Competência Cultural e Contexto: Instituições de Ensino de Enfermagem). Esta mnemónica é fundamental para organizar a seleção dos estudos, extração de dados, mapear a evidência e explicar o seu significado ${ }^{14}$.

A partir da mnemónica PCC supracitada, formulouse a seguinte Questão de Pesquisa: Quais são as estratégias de desenvolvimento da CC durante a formação dos estudantes nas Instituições de Ensino de Enfermagem? Assim, definiu-se o seguinte objetivo, identificar e mapear as estratégias de desenvolvimento da competência cultural durante a formação dos estudantes nas Instituições de Ensino de Enfermagem. 
Desenvolvimento da competência cultural na formação dos estudantes de enfermagem Jose EAR, Coelho AMSRM, Gouveia MC, Paiva MG, Brito IS, Berardinelli LMM, Vicente CMFB

Organizar a seleção dos estudos e extração de dados exige a definição de critérios. Portanto, para manter a coerência entre a questão de revisão, o planejamento e a escolha da estratégia de pesquisa foram definidos, de modo claro e objetivo, os critérios de inclusão e exclusão ${ }^{15}$. A inclusão se baseou em estudos com texto integral disponível; estudos que incluem os estudantes de enfermagem; estudos que analisam as diversas estratégias de desenvolvimento da CC, que contribuam para a aquisição de conhecimentos; estudos que abordem as estratégias do desenvolvimento da CC, realizadas no contexto das Instituições de Ensino de Enfermagem, incluindo aulas de tipologia teórica ou teórico práticas ou práticas, lecionadas aos estudantes durante a licenciatura de enfermagem; estudos primários de investigação; estudos de métodos quantitativos, qualitativos e mistos; nos Idioma português, inglês e espanhol. Os de exclusão são estudos de opinião; estudos epidemiológicos; artigos duplicados; artigos cujo conteúdo não se relacione com o fenómeno de interesse nem com os objetivos deste estudo.

A pesquisa da evidência científica foi realizada, em maio de 2021, com recurso aos motores de busca EBSCOhost e PubMed, e nas bases de dados Cumulative Index to Nursing and Allied Health Literature e MedLine. Para a realização da pesquisa, nas plataformas, recorremos às palavras-chave e termos de indexação "cultural competence", "cultural awareness", "cultural competency", "cultural sensivity", "intercultural competence", "multicultural competence", "nursing education", "nurse education", "continuing education", "training program", "training", "nursing instruction", "strateg*", "methods" $e$ "techniques". Os MeSH terms e os CINAHL subject headings utilizados foram: "continuing nursing education"; "cultural competence" $e$ "learning methods".

Através da pesquisa com a aplicação da combinação definida, obteve-se um total de 890 artigos, sendo que após a aplicação dos critérios de inclusão referidos anteriormente, o total reduziu para 50 resultados, excluindo-se 415 artigos das bases de dados MedLine e 422 da CINAHL, com recurso ao software Rayyan QCRI. Em seguida procedeu-se à eliminação de artigos repetidos e leitura do título, tendo sido excluídos 8 artigos repetidos. Após a leitura do resumo excluíram-se ainda 28 artigos, ficando uma amostra de 15 artigos, que, após leitura integral do texto, excluíram-se 8 , ficando 7 artigos que se verificou serem adequados a temática. Todo este processo de seleção está esquematizado na Figura 1 que representa o Fluxograma Prisma.

Figura 1. Fluxograma PRISMA de seleção dos estudos. Porto, Portugal, 2021

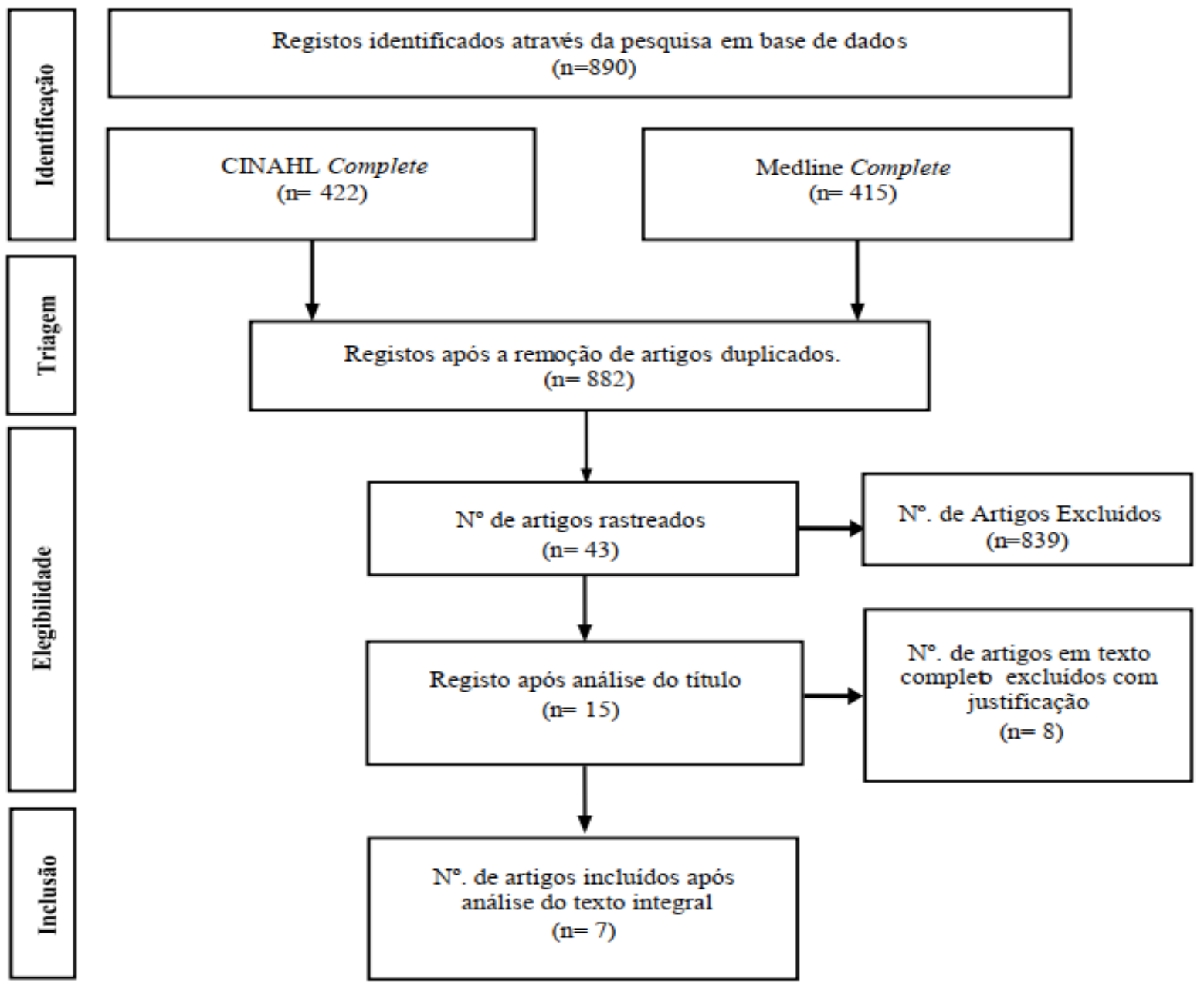




\section{Resultados}

Realizando a síntese dos estudos selecionados, com o objetivo de identificar e mapear as estratégias de desenvolvimento da competência cultural que contribuem para a formação dos estudantes de Enfermagem, encontramos 7 artigos, a saber: três artigos dos Estados Unidos da América (EUA1, EUA2, EUA3) ${ }^{16-17}$, um artigo do Japão (JP1) ${ }^{19}$, dois artigos de Taiwan (TW1, TW2) ${ }^{20,21}$ e um da Turquia (TR1) 22 , publicados entre 2012 e 2020. Para facilitar a apresentação, os estudos serão identificados pelas suas respectivas siglas.

Os participantes nos estudos são estudantes de Enfermagem e de outras áreas da saúde (EUA3) e Professores de Enfermagem (EUA1). Nos estudos que se focam nos estudantes de Enfermagem, quatro referem o ano de curso, onde participaram grupos de jovens dos anos
Desenvolvimento da competência cultural na formação dos estudantes de enfermagem ose EAR, Coelho AMSRM, Gouveia MC, Paiva MG, Brito IS, Berardinelli LMM, Vicente CMFB iniciais do curso (TR1 e TW2), no último ano (EUA2) e de todos os anos (JP1). Quanto aos modelos que fundamentam os estudos, um refere-se ao Modelo Sunrise de Leininger, ao modelo de cuidado culturalmente competente de Campinha- Bacote e ao modelo de competência cultural em enfermagem de saúde mental e psiquiátrica (TW2), um refere o Modelo de Desenvolvimento de Sensibilidade Intercultural de Bennett e o Modelo de Processo de Competência Intercultural de Deardorff (EUA3) e, por último, um refere o Modelo Teórico da Diversidade e Universalidade do Cuidado Cultural de Leininger (TW1).

O Quadro 1 apresenta os artigos em relação ao título, autor, ano de publicação, país, objetivo, tipo de estudo e base de dados. De modo a organizar a exposição dos dados, os artigos foram codificados com letras e números, de acordo com o país onde foi realizado o estudo, listados por ordem alfabética.

Quadro 1. Categorização dos estudos incluídos na revisão. Porto, Portugal, 2021

\begin{tabular}{|c|c|c|c|c|c|}
\hline Título & Autor & Ano & País/Sigla & Objetivo (s) & Tipo de Estudo \\
\hline $\begin{array}{l}\text { Teaching Strategies } \\
\text { that Promote } \\
\text { aculturally Sensitive } \\
\text { Nursing Education }\end{array}$ & Dewald R. & 2012 & $\begin{array}{l}\text { Estados } \\
\text { Unidos da } \\
\text { América } \\
\text { EUA1 }\end{array}$ & $\begin{array}{l}\text { Explorar estratégias de ensino que } \\
\text { promovam uma educação e } \\
\text { cuidados de enfermagem } \\
\text { culturalmente sensíveis. }\end{array}$ & $\begin{array}{c}\text { Estudo descritivo, } \\
\text { qualitativo com técnica Delphi. }\end{array}$ \\
\hline $\begin{array}{l}\text { Being the stranger: } \\
\text { Comparing study } \\
\text { abroad experiences of } \\
\text { nursing students in } \\
\text { low- and high-income } \\
\text { countries through } \\
\text { hermeneutical } \\
\text { phenomenology }\end{array}$ & $\begin{array}{l}\text { Maltby H, } \\
\text { Vries-Erich J } \\
\text { \& Lund } \mathrm{K} \text {. }\end{array}$ & 2016 & $\begin{array}{l}\text { Estados } \\
\text { Unidos da } \\
\text { América } \\
\text { EUA2 }\end{array}$ & $\begin{array}{c}\text { Explorar as experiências dos } \\
\text { estudantes de enfermagem } \\
\text { americanos que realizaram } \\
\text { mobilidade no exterior, num país } \\
\text { considerado subdesenvolvido } \\
\text { (Bangladesh) e num país } \\
\text { considerado desenvolvido } \\
\text { (Holanda). }\end{array}$ & $\begin{array}{l}\text { Estudo qualitativo, } \\
\text { fenomenológico } \\
\text { hermenêutico. }\end{array}$ \\
\hline $\begin{array}{c}\text { Intercultural } \\
\text { competency } \\
\text { development of health } \\
\text { professions students } \\
\text { during study abroad in } \\
\text { India }\end{array}$ & $\begin{array}{l}\text { Doorenbos A } \\
\text { \& Richards C. }\end{array}$ & 2016 & $\begin{array}{l}\text { Estados } \\
\text { Unidos da } \\
\text { América } \\
\text { EUA3 }\end{array}$ & $\begin{array}{l}\text { Descrever e avaliar a eficácia de } \\
\text { um Programa de Mobilidade de } \\
\text { 3semanas da área da saúde e um } \\
\text { currículo de competência } \\
\text { intercultural no aumento das } \\
\text { habilidades e conhecimento dos } \\
\text { estudantes. }\end{array}$ & Estudo misto, pré e pós teste. \\
\hline $\begin{array}{l}\text { Learning experiences } \\
\text { and identity } \\
\text { development of } \\
\text { Japanese nursing } \\
\text { students through } \\
\text { study abroad: } a \\
\text { qualitative analysis }\end{array}$ & $\begin{array}{c}\text { Asahara } \\
\text { K,Huffman J,Inoue } \\
\text { M,Nagai } \\
\text { T,Nakajima } \\
\text { K.Oguro M,Okubo } \\
\text { N,Saitoh } \\
\text { A,Shimoda } \\
\text { K,Tashiro J,Umeda } \\
\text { M \&Uriuda M. }\end{array}$ & 2020 & $\begin{array}{l}\text { Japão } \\
\text { JP1 }\end{array}$ & $\begin{array}{c}\text { Analisar as experiências e } \\
\text { percepções de estudantes de } \\
\text { enfermagem de uma Universidade } \\
\text { no Japão que realizaram um } \\
\text { programa de mobilidade na Ásia e } \\
\text { na América do Norte, identificando } \\
\text { o seu benefício. }\end{array}$ & $\begin{array}{l}\text { Estudo qualitativo, } \\
\text { dedutivo e indutivo. }\end{array}$ \\
\hline $\begin{array}{c}\text { Effects of } \\
\text { Transcultural Nursing } \\
\text { Education on the } \\
\text { Professional Values, } \\
\text { Empathic Skills, } \\
\text { Cultural Sensitivity } \\
\text { and Intelligence of } \\
\text { Students }\end{array}$ & $\begin{array}{l}\text { Kaçan C \& } \\
\text { Örsal Ö. }\end{array}$ & 2020 & $\begin{array}{l}\text { Turquia } \\
\text { TR1 }\end{array}$ & $\begin{array}{l}\text { Examinar o efeito da educação } \\
\text { transcultural nos valores } \\
\text { profissionais, habilidades } \\
\text { empáticas, sensibilidade cultural e } \\
\text { inteligência dos estudantes de } \\
\text { enfermagem. }\end{array}$ & $\begin{array}{l}\text { Estudo quasi-experimental } \\
\text { com grupo } \\
\text { de controle, pós-teste. }\end{array}$ \\
\hline $\begin{array}{l}\text { Cultural competence } \\
\text { course for nursing } \\
\text { students in Taiwan: A } \\
\text { longitudinal study }\end{array}$ & $\begin{array}{l}\text { Chang } P, \\
\text { Huang } M, \text { Lin C \& } \\
\text { Wang, L. }\end{array}$ & 2015 & $\begin{array}{l}\text { Taiwan } \\
\text { Tw1 }\end{array}$ & $\begin{array}{l}\text { Avaliar os efeitos deum curso de } \\
\text { CC para estudantes de } \\
\text { enfermagem, de duas } \\
\text { Universidades distintas em Taiwan. }\end{array}$ & $\begin{array}{l}\text { Estudo longitudinal, } \\
\text { quasi-experimental, pré-teste, } \\
\text { pós-teste e follow-up. }\end{array}$ \\
\hline $\begin{array}{l}\text { Evaluation of nursing } \\
\text { students' perceptions } \\
\text { of their cultural care } \\
\text { competency: A mixed } \\
\text { method study in } \\
\text { Taiwan }\end{array}$ & $\begin{array}{l}\text { Chen Y., Hung CC., } \\
\text { Liang H., } \\
\text { Wang, Y \& } \\
\text { Wu K. }\end{array}$ & 2019 & $\begin{array}{l}\text { Taiwan } \\
\text { Tw2 }\end{array}$ & $\begin{array}{l}\text { Avaliar como a implementação de } \\
\text { um curso de CC, inserido num } \\
\text { curso de cuidados de enfermagem } \\
\text { acrianças e adolescentes, afetou a } \\
\text { percepção dos estudantes sobre a } \\
\text { sua própria competência cultural. }\end{array}$ & $\begin{array}{c}\text { Estudo explicativo, } \\
\text { progressivo de } \\
\text { abordagem mista, pré e pós- } \\
\text { teste. }\end{array}$ \\
\hline
\end{tabular}


Desenvolvimento da competência cultural na formação dos estudantes de enfermagem Jose EAR, Coelho AMSRM, Gouveia MC, Paiva MG, Brito IS, Berardinelli LMM, Vicente CMFB

A seguir, apresentamos os tipos de estratégias de desenvolvimento da CC evidenciadas nos estudos (Quadro 2). Usaremos siglas para facilitar a análise: Unidades curriculares de tipologia teórica (T), teórico-prática (TP), prática laboratorial (PL) e ensaio ensino clínico (EC).

\begin{tabular}{|c|c|}
\hline Estudo & Estratégia \\
\hline EUA1 & $\begin{array}{l}\text { Em geral: } \\
\text { Integrar a sensibilidade cultural em todos os cursos e incluir perspectivas culturais de cuidar no currículo; Abordagem de modelos } \\
\text { de CC; trabalhar com estudantes individualmente para superar as barreiras de aprendizagem. } \\
\text { Unidades curriculares: T, TP e PL: Ensinar a respeitar a dignidade humana, a utilizar linguagem compreensível, transmitir empatia } \\
\text { genuína, recorrer a análises de casos, cenários e/ou roleplay; Seminários e Workshops. } \\
\text { EC: Contacto com utentes de diversas culturas; Fornecer tutoria e orientação, recorrendo a tutores culturalmente sensíveis; } \\
\text { promover o pensamento crítico, incentivando os estudantes a analisar atitudes, crenças e reações; incentivar a autoconfiança, } \\
\text { promover a autoeficácia; Evitar generalizações e estereótipos. }\end{array}$ \\
\hline EUA2 & $\begin{array}{l}\text { Unidades Curriculares: T: Aulas teóricas integradas no Curso de Saúde Pública. } \\
\text { EC: Visita a Instituições de Cuidados de Saúde; Realização de estudos da comunidade, através de visitas de campo; Visitas a locais } \\
\text { históricos; Experienciar o dia a dia em várias comunidades. }\end{array}$ \\
\hline EUA3 & $\begin{array}{l}\text { Unidades Curriculares: T: Sessões de preparação e orientação pré partida; participação numa discussão de grupo; Planos de } \\
\text { desenvolvimento pessoal; Elaboração de reflexões guiadas, com base no modelo de desenvolvimento de sensibilidade } \\
\text { intercultural de Bennett e no modelo de processo de competência intercultural de Deardorff. } \\
\text { EC: Experiência cultural e prática clínica rotativa em dois hospitais (Hospital público e privado) nas diversas áreas clínicas (saúde } \\
\text { comunitária, psicologia, medicina familiar, reabilitação, cirurgia, internamento de cirurgia médica, urgência pediátrica, } \\
\text { internamento de medicina e farmácia) - pós-teste. }\end{array}$ \\
\hline JP1 & $\begin{array}{l}\text { Unidades Curriculares T: Sessões de orientação e Preparação de Apresentações em Inglês, antes do Programa de Mobilidade; } \\
\text { Aprender sobre Inglês técnico aplicado à prática de Enfermagem, no Canadá; Desenvolver habilidades comunicacionais em Inglês, } \\
\text { no Canadá; Aprender sobre questões de saúde comunitária sob uma perspectiva global, nos EUA; Aprender sobre sistemas de } \\
\text { saúde e práticas de enfermagem, na Tailândia e na Coreia do Sul; Aprender sobre medicina chinesa e cuidados de saúde, em } \\
\text { Taiwan; Textos de reflexão após o programa. } \\
\text { EC: Cultivar uma perspectiva global integrando cursos de enfermagem nos EUA; Prática de enfermagem em países considerados } \\
\text { desenvolvidos e subdesenvolvidos; realizar serviços de voluntariado, nas Filipinas; }\end{array}$ \\
\hline TR1 & $\begin{array}{l}\text { Unidades Curriculares: T: Convidar diferentes oradores para debater problemas culturais; Relatos de testemunhos; Assistir a } \\
\text { filmes, interpretar artigos e discutir em grupo; discutir aspetos positivos e negativos de experiências sociais individuais. } \\
\text { PL: Casos práticos encenados pelos estudantes. }\end{array}$ \\
\hline TW1 & $\begin{array}{l}\text { Unidade Curricular T: Assistir a palestras; Apresentações de grupo baseadas no Modelo Teórico da Diversidade e Universalidade } \\
\text { do Cuidado Cultural, de Leininger; Visualizar vídeos e posterior discussão deles; Reflexões pessoais }\end{array}$ \\
\hline TW2 & $\begin{array}{l}\text { Unidades Curriculares: T: Modelo Sunrise de Leininger, Modelo de Cuidado Culturalmente Competente e Modelo de CC em } \\
\text { Enfermagem de Saúde Mental e Psiquiátrica, de Campinha- Bacote; Ensino didático sobre cuidados culturalmente } \\
\text { competentes; Opinião sobre o curso. } \\
\text { PL: Apresentação de cenários sobre conflitos culturais com o objetivo de estimular o pensamento crítico. }\end{array}$ \\
\hline
\end{tabular}

\section{Discussão}

Nos artigos em estudo, os tipos de estratégias mais evidenciadas para o desenvolvimento da CC foram programas de mobilidade (EUA2, EUA3 e JP1) e cursos de ensino-aprendizagem da CC, com a duração máxima de um semestre (TR1, TW1 e TW2).

É de realçar que dos sete artigos selecionados, apenas um (EUA1) destaca os professores como especialistas sobre as melhores práticas que promovem a sensibilidade cultural no Ensino de Enfermagem, apesar de descreverem diversas formas de estimular o ensinoaprendizagem da CC.

\section{Programas de mobilidade}

Os programas de mobilidade são definidos como qualquer experiência educacional que envolva uma pessoa, geralmente um estudante, que deixa o seu país de origem por um determinado período. Essa experiência pode ser organizada e oferecida por uma universidade ou o estudante pode planear e envolver-se nela por iniciativa própria. Os programas de mobilidade podem ser de curto prazo, de uma a quatro semanas, ou longo prazo, mais de quatro semanas, e podem envolver experiências em países considerados desenvolvidos ou subdesenvolvidos ${ }^{19}$. Os estudos que abordam programas de mobilidade, nomeadamente EUA2, EUA3 e JP1, apresentam alguns aspetos convergentes e divergentes. Os três programas de mobilidade contemplam componentes teóricos e práticos. Relativamente os componentes teóricos, os estudos EUA3 e JP1, apresentam em comum sessões de orientação e preparação pré partida, contrariamente ao estudo EUA2, que apenas aborda o curso que desenvolve durante o programa. Ainda no componente teórico, os estudos JP1 e EUA3, englobam reflexões pessoais, que se referem a experiência durante o programa (JP1) e, que se baseiam em modelos de CC (EUA3).

No que concerne ao componente prático destes programas, pode-se constatar que em todos os estudos referentes aos programas de mobilidade, EUA2, EUA3 e JP1, os estudantes contactaram com instituições de saúde. Além do referido, nos estudos EUA2 e EUA3, para que os estudantes conhecessem o meio, a cultura onde estão inseridos, e a sua população, procederam à visita de locais pertinentes para o efeito, tais como, cidades, visitas de campo e estudos da comunidade. Por outro lado, no estudo JP1, os estudantes prepararam apresentações em inglês para facilitar a sua adaptação, em termos linguísticos, ao local onde iriam realizar o programa.

Analisando os efeitos do aumento de CC nos estudantes em programas de mobilidade (EUA2, EUA3 e JP1), os resultados sugeriram que todos os estudantes alteraram a sua visão do mundo e desenvolveram a sua consciência cultural, independentemente de terem uma 
Desenvolvimento da competência cultural na formação dos estudantes de enfermagem Jose EAR, Coelho AMSRM, Gouveia MC, Paiva MG, Brito IS, Berardinelli LMM, Vicente CMFB

experiência num país considerado subdesenvolvido ou desenvolvido. As experiências dos estudantes durante os programas de mobilidade podem diferir, de acordo com a duração e o destino dos mesmos e se realizam estes programas em grupo ou individualmente ${ }^{23}$.

A preparação pré-partida é essencial para orientar e preparar os estudantes para que os objetivos sejam atingidos. Os estudantes necessitam de apoio, antes, durante e após a sua experiência internacional, não apenas com a partilha de informações, mas, também, através de sessões apropriadas de reflexão e esclarecimento. Além disso, os docentes que supervisionam estudantes em programas de mobilidade necessitam de compreender os impactos positivos desta experiência na aquisição de competências, incentivando-os a compreender o significado das suas experiências de aprendizagem. Os programas de mobilidade são um recurso valioso para que os estudantes e profissionais estejam melhor preparados para a prática de enfermagem no mundo globalizado ${ }^{23}$.

\section{Cursos de CC}

Todos os estudos, TR1, TW1 e TW2, abordaram modelos relacionados com a cultura. No estudo TW1, realizaram apresentações de grupo sobre modelos de CC, nomeadamente o Modelo Teórico da Diversidade e Universalidade do Cuidado Cultural de Leininger, no estudo TW2 recorreram ao Modelo de Sunrise de Leininger, Modelo de Cuidado Culturalmente Competente e ao Modelo de CC em Enfermagem de Saúde Mental e Psiquiátrica, de Campinha-Bacote e no estudo TR1 há referência na utilização de Modelos Transculturais de Enfermagem, durante o curso, porém não estão identificados.

Os resultados dos três estudos que abordam cursos de CC (TR1, TW1 e TW2) revelam a eficácia deles. No artigo TW1, a CC de todos os participantes melhorou na avaliação pós-teste, constataram-se ainda melhor resultados nos estudantes que participaram no curso (grupo experimental), do que nos estudantes que não participaram (grupo controle), confirmando que realizar um curso de CC aumenta efetivamente as competências dos estudantes. Do mesmo modo, o estudo TW2 também mostrou melhores resultados na avaliação pós-teste, uma vez que houve um aumento dos conhecimentos, atitudes e habilidades dos cuidados culturalmente competentes, após o curso de CC. No estudo TR1, as pontuações do grupo experimental foram mais elevadas do que as do grupo de controlo, neste estudo, o curso de Enfermagem Transcultural apresentou-se como um método eficaz, nomeadamente a nível da sensibilidade cultural, empatia, inteligência cultural e valores profissionais.

Por último, o estudo EUA1, identifica as estratégias e práticas de ensino recomendadas para a educação de enfermagem, na perspectiva de especialistas da área. Os tipos de estratégias foram hierarquizados de acordo com a sua relevância. Os especialistas em estudo, afirmam que o uso destas estratégias potencializa o conhecimento dos estudantes em CC. Algumas das estratégias mencionadas neste estudo, vão de encontro às estratégias evidenciadas nos estudos anteriormente abordados. Tais como, abordagem de modelos de CC (EUA3, TW1 e TW2), recurso a análise de casos, cenários e role-play (TR1 e TW2), promover o pensamento crítico dos estudantes (EUA3, TR1 e TW1), contato com utentes de diversas culturas (EUA2, EUA3 e JP1) e, ainda, seminários sobre CC (EUA3).

Todos os autores concluíram que os programas de estudos das escolas de enfermagem precisam ser revistos, de modo que todos os estudantes tenham as mesmas oportunidades de adquirir a $\mathrm{CC}$ e, consequentemente, exista uma melhoria dos cuidados de saúde.

Os modelos curriculares existentes, muitas vezes, não respondem as necessidades dos enfermeiros que contactam com diversos pacientes em diversos contextos clínicos. A mudança de paradigma exigirá mudanças fulcrais no modo como os docentes de enfermagem consideram, organizam e fornecem o conteúdo cultural para o desenvolvimento da CC dos estudantes. As estratégias tradicionalmente usadas na educação, podem não ser suficientes para atender de imediato necessidade de resposta à diversidade cultural dos pacientes nos cuidados de saúde ${ }^{24}$

A intenção de um currículo que contemple estratégias de desenvolvimento de CC, é garantir que os estudantes detenham o conhecimento, as atitudes e as habilidades que lhes permitam trabalhar efetivamente com os pacientes e suas famílias, bem como com outros membros da comunidade e das equipes multidisciplinares de saúde ${ }^{24,25}$, considerando a cultura de todos.

\section{Conclusão}

O número crescente de pacientes de diversas culturas cria um enorme desafio para os enfermeiros, exigindo um cuidado individualizado e holístico baseado nas necessidades culturais de cada pessoa. Isso requer dos enfermeiros o conhecimento e a compreensão das diferentes culturas relativamente à saúde, crenças, valores e costumes. O conhecimento transcultural é importante para os enfermeiros, para que estes se tornem sensíveis às necessidades de indivíduos de várias culturas. Destaca-se que estes profissionais têm um contato próximo com os pacientes e são responsáveis pela formulação de planos de cuidados a fim de satisfazer as necessidades individuais dos pacientes.

A aquisição de Competência Cultural, nos estudantes de enfermagem e enfermeiros, é uma medida fulcral para a diminuição das disparidades e garantir a universalidade, integralidade e equidade nos serviços de saúde. O desenvolvimento da CC durante a formação dos estudantes deve ser uma prioridade. Embora a educação, por si só, seja provavelmente insuficiente para melhorar a CC é imperativo que haja uma abordagem sistemática e contínua para a sua melhoria. Conclui-se que as estratégias para o Desenvolvimento da Competência Cultural, devem fazer parte do processo formativo dos estudantes de enfermagem, pois, a educação intercultural é uma proposta político-pedagógica que vislumbra a formação para a cidadania, a superação de preconceitos e discriminações que expropriam de seus direitos os indivíduos e as comunidades. 
Desenvolvimento da competência cultural na formação dos estudantes de enfermagem Jose EAR, Coelho AMSRM, Gouveia MC, Paiva MG, Brito IS, Berardinelli LMM, Vicente CMFB

\section{Referências}

1. Moita M, Silva A. Modelos de Competência Cultural: Uma Análise Crítica. Pensar Enfermagem. 2016;20(2):72-89.

2. Carvalho A, Coutinho E, Parreira V, Rodrigues E. A competência cultural em enfermagem e a mediação intercultural preventiva. Número Temático Mediação Intercultural, Observatório das Migrações (OM) [Internet]. 2018;15:66-81.

3. International Organization for Migration (IOM). World Migration Report 2020. Geneva: IOM; 2020.

4. Brasil. Lei $\mathrm{n}$. 8080, de 19 de setembro de 1990. Dispõe sobre a organização do SUS. Brasília (DF): Casa Civil; 2011.

5. Brasil. Decreto n.o 7508, de 28 de junho de 2011. Regulamenta a Lei n.o 8080 de 1990. Brasília (DF): Casa Civil; 2013.

6. Barroso D, Araújo JMA. Vade Mecum OAB 2017. Rev., atual e ampl. 2017;9.

7. Portugal. Constituição da República Portuguesa. Revisão Constitucional [Internet]. Portugal; 2005 [acesso em 02 dez 2018 ]. Disponível em: https://www.parlamento.pt/Legislacao/Paginas/ConstituicaoRepublicaPortuguesa.aspx

8. Cabo Verde. Constituição da República de Cabo Verde. Dispõe sobre a Constituição Federal do Cabo Verde [Internet]. Cabo Verde; 1992 [acesso em 02 dez 2018]. Disponível em: https://www.governo.cv/governo/constituicao/

9. McMichael T, Beaglehole R. The global context for public health. In: BEAGLEHOLE R. Global public health: a new era. New York: Oxford University; 2003.

10. Coutinho $\mathrm{E}$, et al. $\mathrm{O}$ cuidado cultural na trajetória da enfermagem transcultural e competência cultural. Congresso lbero-americano de Investigação Qualitativa (CIAQ) [Internet]. 2017 [acesso em $11 \mathrm{dez} 2018$ ];2(6). Disponível em: https://proceedings.ciaiq.org/index.php/ciaiq2017/article/view/1510

11. Carvalho EC, Parreira AC, Castro V. A competência cultural em enfermagem e a Mediação Intercultural preventiva, Número Temático Mediação Intercultural, Observatório das Migrações (OM), Revista Migrações. 2018;15:66-81.

12. Fontana RT. A Interculturalidade Na Formação Dos Profissionais De Enfermagem. Revista Contexto \& Amp; Educação. 2019;34(109):3651. DOI: $10.21527 / 2179-1309.2019 .109 .36-51$

13. Ministério da Educação (BR). Diretrizes Curriculares Nacionais do Curso de Graduação em Enfermagem. [Internet]. Brasília (DF): MEC; 2001. Disponível em: http://portal.mec.gov.br/cne/arquivos/pdf/Enf.pdf

14. Galvão C, Mendes K, Silveira R. Revisão Integrativa: Método de Pesquisa para a Incorporação de Evidências na Saúde e na Enfermagem. Texto Contexto Enferm [Internet]. 2008 [acesso em $11 \mathrm{dez}$ 2018];17(4):758-764. Disponível em: https://www.scielo.br/j/tce/a/XzFkq6tjWs4wHNqNjKJLkXQ/?format=pdf\&lang=pt

15. The Joanna Briggs Institute (JBI). JBI manual for evidence synthesis. 2021. DOI: 10.46658/JBIMES-20-01

16. Apóstolo J. Síntese da evidência no contexto da translação da ciência. Coimbra, Portugal: Escola Superior de Enfermagem de Coimbra (ESEnfC), 2017.

17. Dewald R. Teaching strategies that promote a culturally sensitive nursing education. Nursing Education Perspective. 2012;33(6):410-412. DOI: 10.5480/1536-5026- 33.6.410.

18. Lund K, Maltby H, Vries-EJ. Being the stranger: comparing study abroad experiences of nursing students in low- and high-income countries through hermeneutical phenomenology. Journal of Nurse Education Today. 2016;45(1):114-199. DOI: 10.1016/j.nedt.2016.06.025

19. Doorenbos A, Richards C. Intercultural competency development of health professions students during study abroad in India. Journal of Nursing Education and Practice. 2016;6(12):89-98. DOI: 10.5430/jnep.v6n12p89

20. Asahara K, Huffman J, Inoue M, Nagai T, Nakajima K, Oguro M, Okubo N, Saitoh A, Shimoda K, Tashiro J, Umeda M, Uriuda M. Learning experiences and identity development of japonese nursing students through study abroad: a qualitative analysis. International Journal of Medical Education. 2020;11(1):54-61. DOI: 10.5116/ijme.5e47.cf1b

21. Orsal $\mathrm{O}$, Kaçan C. Effects of transcultural nursing education on the professional values, empathic skills, cultural sensitivy and intelligence of students. Journal of Community Health Nursing. 2020;37(2):65-76. DOI: 10.1080/07370016.2020.1736374

22. Chang $P$, Huang $M$, Lin C, Wang L. Cultural competence course for nursing students in Taiwan: a longitudinal study. Journal of Nurse Education Today. 2015;35(1):1268-1274. DOI: 10.1016/j.nedt.2015.05.023

23. Tricco AC, et al. Extensão PRISMA para análises de escopo (PRISMA-ScR): checklist e explicação. Ann Intern Med. 2018;169(7):467-473. DOI: 10.7326 / M18-0850.

24. Kunaviktiku W, Mesukko J, Turale S. Giving undergraduate nursing students international experiences: Issues and strategies. Nursing \& Health Sciences. 2020;7. DOi:10.1111/12722.

25. Souza RR, Oliveira LL. Revisões integrativas na assistência da Enfermagem Obstétrica e Ginecológica em tempos pandêmicos: desafios e possibilidades. Glob Acad Nurs. 2021;2(Spe.1):e89. DOI: 10.5935/2675-5602.20200089 